\title{
EFFECT OF MAGNETIZATION OF BIODIESEL ON ITS CHEMICAL PROPERTIES AND PERFORMANCE AND EMISSION PARAMETERS OF DIESEL ENGINE
}

\section{DOI : 10.36909/jer.ICIPPSD.15513}

\author{
Ajay Chandravanshi*, Shrikant Pandey, Rakesh Kumar Malviya
}

Department of Mechanical Engineering, Shri Vaishnav Vidyapeeth Vishwavidyalaya Indore, India.

*Email: chandravanshiajay87@gmail.com; Corresponding Author.

\begin{abstract}
Utilization of biodiesel results in lower emissions of hydrocarbons, carbon mono oxide, and smoke. But with the use of biodiesel as fuel emission of Oxides of nitrogen increases along with this some performance parameters also deteriorated. In the present investigation, the fact has been used that, the magnetization of fuels containing hydrocarbon changes the chemical properties as well as orientation and arrangement of molecules of fuel. In this experimental investigation density and calorific values of biodiesel have been determined. In another setup of experimentation on a single-cylinder four-stroke diesel engine, observations are taken with diesel, biodiesel blend containing 20\% biodiesel (BD20), and magnetized biodiesel (BD20+MF) as fuel. Results of this investigation show that, the magnetization of biodiesel is not only helpful in increasing performance parameters like brake specific fuel consumption and brake thermal efficiency but also it helps to control the emissions of carbon mono oxide, oxides of nitrogen, hydrocarbons, and smoke. Maintaining the magnetic field just before the entry of the combustion chamber on the fuel line is a little hard, due to the regular increase in temperature of the spot where magnets are kept. It is possible that, if the strength of the magnetic field is increased then, there may be further improvement in performance and engine parameters, as in this investigation only 4000 Gauss Magnetic field has been used.
\end{abstract}

Key words: Magnetization; Biodiesel; Diesel Engine; Performance Parameters; Emission.

\section{INTRODUCTION}

At present time the fuel used for the internal combustion engine, mainly composed of hydrocarbon molecules (Wahab et al., 2017, Sahoo and Jain, 2019, Faris et al., 2012, Pramod Kumar et al. 2017). These hydrocarbon fuels are fossil in nature and the price of these fuels is increasing day by day. One of the reasons for the increasing price may be the depletion of 
fossil fuel resources (Afzal et al., 2018). In this situation, we will have to look for some alternate fuel and at the same time higher performance rate with lower cost. Many efforts of research have been done in the search of alternate fuel and biodiesel is proving to be a great alternate source of energy in a diesel engine.

Biodiesel is not only an alternate source of energy but also a green source of energy, because of lower emissions as compared to conventional fuel. Properties of biodiesel are not the same as that of petroleum fuel, despite that biodiesel can be used in form of blends with different percentages of biodiesel in a diesel engine without engine modification. With lower emission, the limitations of using biodiesel are lower performance parameters (Afzal et al., 2018). To improve performance parameters, there are various techniques used in the diesel engine. In this experimental study, the magnetization of biodiesel has been done to improve performance parameters just before entering the fuel in the combustion chamber.

It is seen that the fuels used in internal combustion engines are in the form of liquid, and do not take part in combustion if they are not atomized. Each molecule is composed of a definite number of atoms comprising of the number of electrons and neutrons. These molecules always have properties of electric charges (Negative or Positive) and magnetic movement. When fuel is fed to the combustion chamber, then the fuel molecules are neither blended properly with oxygen nor realigned. The fuel molecules, as well as chains of hydrocarbon, should be realigned with oxygen and ionized properly for better combustion of fuel. The ionization and realignment are achieved by the application of a magnetic field on the fuel line just before the entry of the combustion chamber. Due to magnetization of fuel structure of hydrocarbon molecules is converted from bundles to small groups having larger space to be interlocked with combustion oxygen and hence improving quality of combustion (Wahab et al., 2017, Sahoo and Jain, 2019, Faris et al., 2012, Pramod Kumar et al. 2017). According to Van Der Waal, the bond between hydrocarbon and oxygen becomes stronger due to the presence of a magnetic field (Wahab et al., 2017). Due to better combustion, the performance parameter like fuel consumption is reduced while emissions like carbon mono oxides, oxides of nitrogen, and hydrocarbon are reduced. Negative and positive charges of hydrogen atoms are due to electron and proton respectively and hydrogen atom has also a property of dipole moment. Hydrogen can be diamagnetic or paramagnetic depending on the rotation of the nucleus under the influence of the magnetic field. So hydrogen atomic orientation takes place in two isomeric forms, one is ortho and the other is para as shown in figure 1 (Wahab et al., 2017, Sahoo and Jain, 2019, Pramod Kumar et al. 2017). 


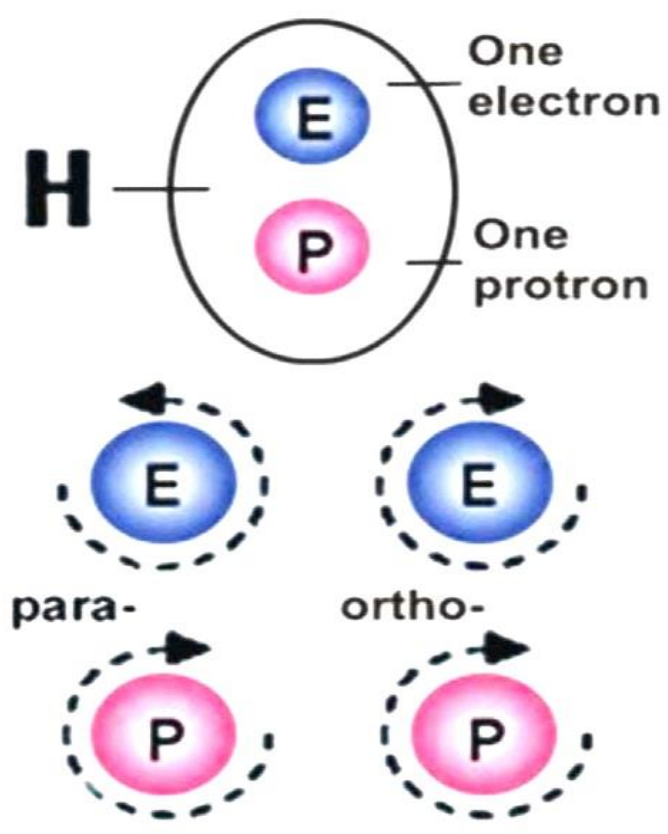

Figure 1 Atomic Orientation (Wahab et al., 2017, Pramod Kumar et al. 2017).

Anti-parallel rotation of nucleus spin is shown by para hydrogen while parallel rotation of nucleus spin is shown by ortho hydrogen as shown in figure 1 and figure 2 .

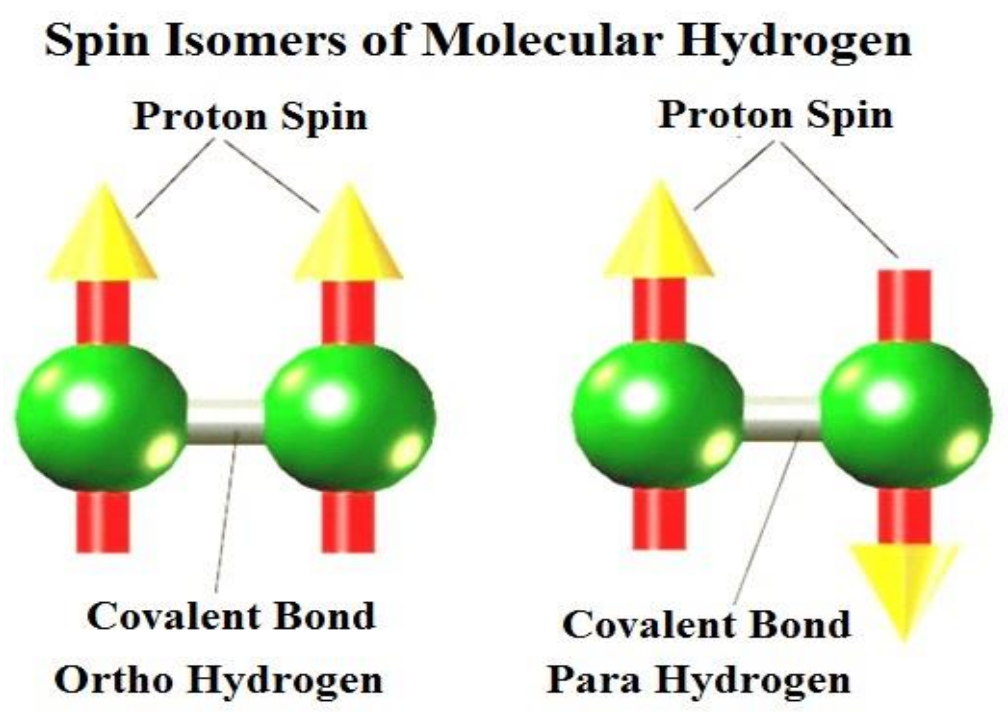

Figure 2 Ortho and Para Hydrogen (Barna and Lelea, 2017)

Ortho hydrogen is in the state of higher energy while parahydrogen is in the state of lower energy. In thermal equilibrium at room temperature hydrogen is composed of $25 \%$ para hydrogen and $75 \%$ ortho hydrogen. The percentage of ortho hydrogen in the fuel atoms can be 
increased by applying a magnetic field along the fuel line (Sahoo and Jain, 2019, Faris et al., 2012, Pramod Kumar et al. 2017).

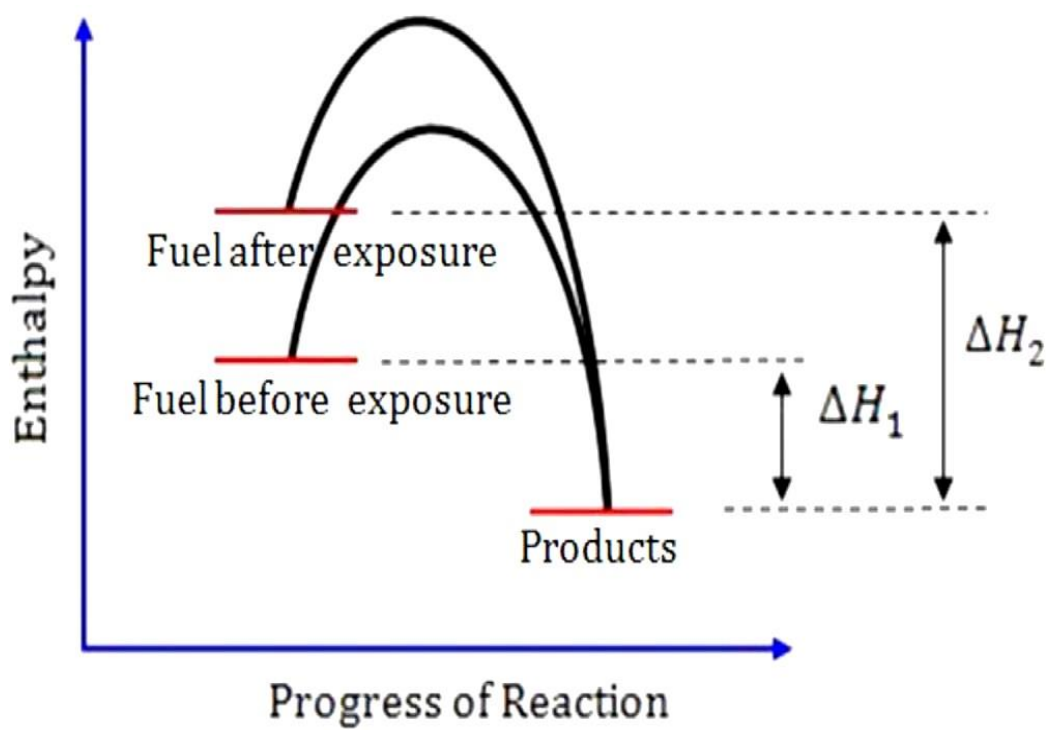

Figure 3 Effect of Magnetization on Combustion Enthalpy (Wahab et al., 2017)

A schematic diagram is shown in the figure 3 to show the effect of magnetization of fuel on combustion enthalpy. The free energy is unchanged because primary bonds present in the atoms have been broken already for building new bonds in the combustion process, thus increasing the enthalpy of combustion due to better quality of fuel and hence reducing the fuel consumption (Wahab et al., 2017, Sahoo and Jain, 2019, Faris et al., 2012, Pramod Kumar et al. 2017).

\section{METHODOLOGY}

How magnetization affects the arrangements and properties of molecules is shown in figure 4. It is seen in the figure that, when fuel is not magnetized then fuel molecules are very close to each other and in cluster form. So, when the fuel is burnt, there is not enough oxygen to get interlocked with the hydrocarbon particles and hence incomplete combustion occurs. But when Magnetization of fuel is done then atoms of hydrocarbons are arranged in a dispersed manner and provide enough space for combustion oxygen and hence results in complete combustion. Methodologies and results which have been applied by various researchers have also been considered in the present investigation and explained below.

It was notified that, the utilization of biodiesel as fuel along with the application of a magnetic field on fuel could be beneficial in the enhancement of fuel burning in the internal combustion engine. Due to improved burning heat energy per unit quantity coming out due to combustion 
is increased with a lower level of hazardous gases outflow with lower specific fuel consumption (Wahab et al., 2017).

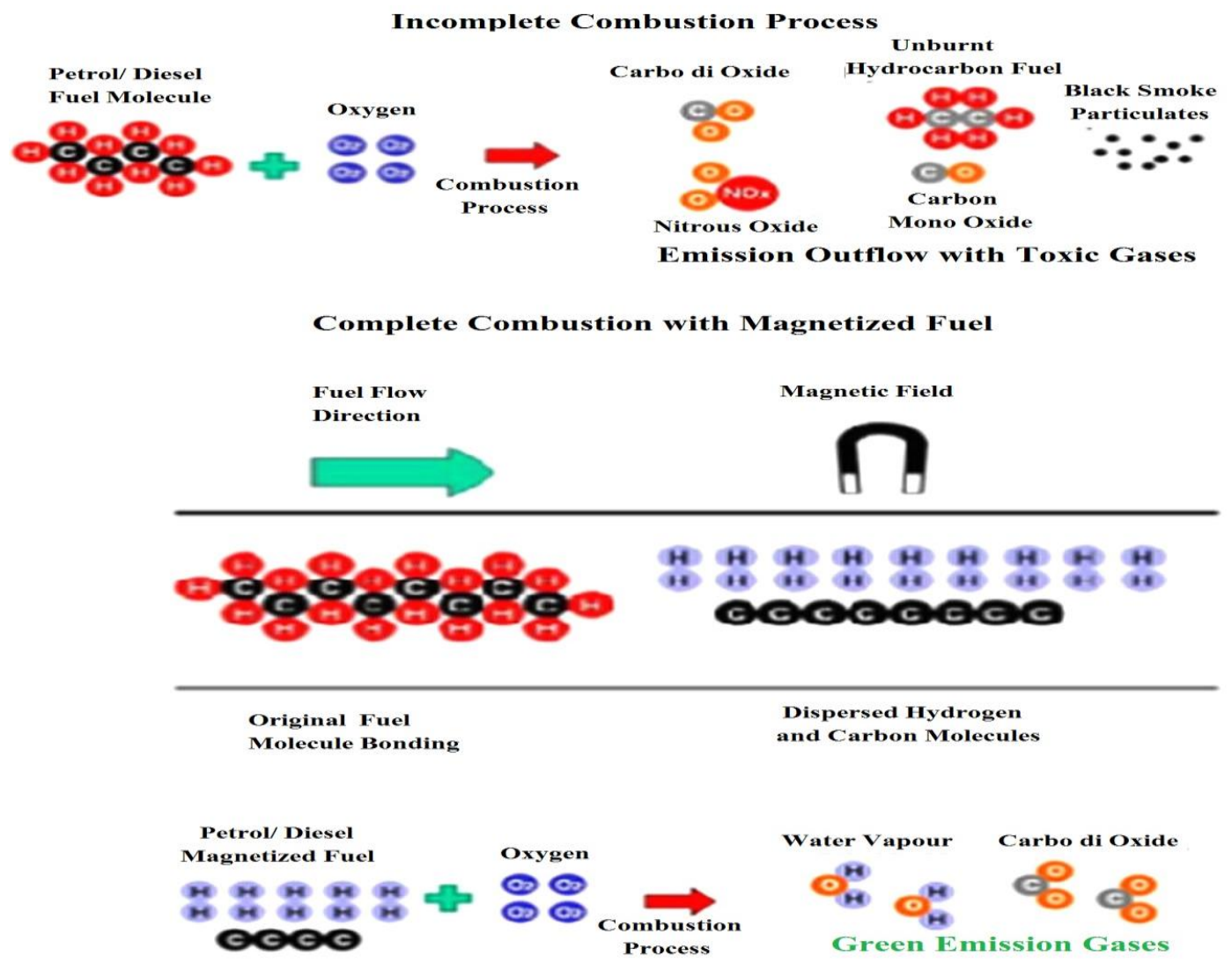

Figure 4 Effect of Magnetic Field on arrangements of molecular arrangements (Wahab et al., 2017)

The mechanical efficiency was found to be $7 \%$ higher, along with this brake specific fuel consumption was $6 \%$ lower, emission of $\mathrm{CO}_{2}$ was $13 \%$ lower and emission of NOx was $19 \%$ lower with the use of $\mathrm{CuO}$ nano fuel in diesel with the application of the magnetic field of 3000 Gauss on fuel line of diesel engine (Sahoo and Jain, 2019).

Karanja oil methyl ester (Karanja Biodiesel) with $20 \%$ methanol was used as fuel with the magnetization of fuel-blends in a diesel engine at full load. Results showed that, $\mathrm{CO}_{2}$ emission, NOx emission, and smoke outflow were $68 \%, 48 \%$, and $51 \%$ lower respectively than that with Karanja biodiesel only (Thiagarajan et al., 2018). Utilization of magnetized fuel in a supercharged diesel engine having low injection pressure with a speed of 2100 RPM to 2400 RPM delivered higher engine torque and brake power and lower while smoke outflow (Barna and Lelea, 2017). Emissions of carbon mono oxides and hydrocarbon through the exhaust 
manifold of diesel engine were found to decrease by $30 \%$ and $40 \%$ respectively, with the use of fuel magnetization of 2000, 4000, 6000 and 9000 Gauss, before entering the combustion chamber, while $\mathrm{CO}_{2}$ emission increases marginally (Faris et al., 2012).

Applying magnetic fields of strengths 4000 to 8000 Gauss, just before carburetor in petrol engine reduced the emissions of carbon dioxides, hydrocarbons, and nitrogen oxides by $30.57 \%, 97 \%$, and $36.08 \%$ respectively, while emission of carbon mono oxides is slightly higher (Pramod Kumar et al., 2017). Magnetization of fuel with a magnet of strength 9000 Gauss before entering the combustion chamber of spark ignition engine improved the quality of combustion of fuel and rate of combustion. This research results in 4 to $12 \%$ lower fuel consumption as compared to without magnetization. Magnetization also reduces carbon mono oxides, Carbon dioxides, hydrocarbons, and oxides of nitrogen up to $11 \%, 10 \%, 18 \%$, and $10 \%$ respectively (Niaki et al., 2019). The magnetization of fuel can bring the fuel consumption down up to $15.5 \%$ at operating condition and reduction in exhaust emission can also be brought down up to $50 \%$ depending upon the strength of the magnet and other operating conditions in a gasoline engine (Rehim and Attia, 2014).

In this investigation, all the observations were taken on a single-cylinder, four-stroke, direct injection, compression ignition engine. The fuel used in this study was Jatropha biodiesel and properties (Density and Calorific Value) of biodiesel were evaluated experimentally and compared the same for with and without magnetization. Various observations were taken on the diesel engine to get performance parameters and different loads and again compared for with and without magnetization of fuel. The magnetization of fuel was done with the help of a permanent magnet of strength 4000 Gauss.

\section{RESULTS AND DISCUSSION}

Various Observations were taken in this study on a four-stroke diesel engine, using biodiesel and diesel blends as fuel. Fuel Blends have been magnetized by putting magnets of 4000 Gauss, just before the entering of fuel in the combustion chamber. Various performance parameters have been calculated with the help of observations and emission parameters have been observed and noted. All the results are discussed as below:

Comparison of properties of fuel after and before magnetization is shown in table 1.

Table 1 Properties of fuel

\begin{tabular}{|l|l|l|l|}
\hline Biodiesel & Without magnet & With magnet & Result \\
\hline
\end{tabular}




\begin{tabular}{|l|c|c|c|}
\hline Density $(\mathrm{kg} / \mathrm{m} 3)$ & 852.12 & 838.44 & $1.605 \%$ reduction \\
\hline Calorific value $(\mathrm{kj} / \mathrm{kg})$ & 41832 & 42308.55 & $1.139 \%$ increment \\
\hline
\end{tabular}

Table 1 shows that after magnetization the density of biodiesel reduces, while the calorific value of biodiesel increases. There is a $1.605 \%$ reduction in the density of biodiesel and a $1.139 \%$ increment in calorific value. This implies that the combustion of magnetized fuel will give more energy than the combustion of biodiesel without magnetization.

\section{PERFORMANCE PARAMETERS}

In this investigation performance parameters considered are mainly brake power (BP), brake specific fuel consumption (BSFC), and brake thermal efficiency (B TE). The effect of biodiesel and magnetic field (MF) on each parameter are discussed and compared with that of diesel only. The fuels used in this study were diesel (D) and the blend of $80 \%$ diesel and $20 \%$ biodiesel (BD20).

\section{Brake Power:}

It is seen in figure 5, that relationship between load and brake power is linear, which is true, but brake power is reduced slightly with BD20. Brake power with BD20+MF was a little higher than that with BD20.

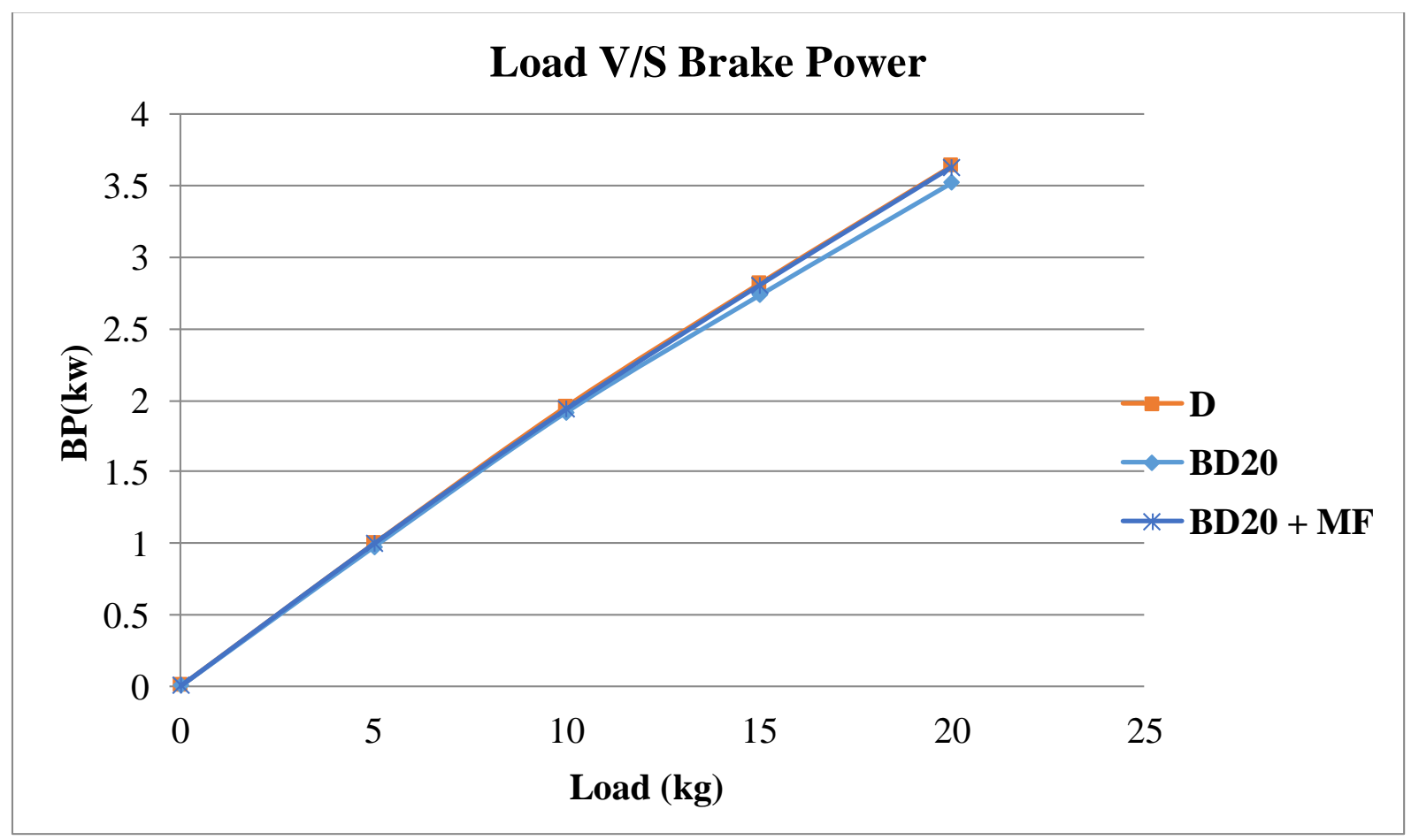

Figure 5 Variation in brake power with load. 
It is observed that the maximum reduction in brake power with BD20 is 3.25\% as compared that with diesel at a higher load. The decrease in brake power is due to the less torque generation with biodiesel as a fuel. Utilization of magnetic field converted this reduction into an increment of $0.17 \%$ as compared that with diesel. This increase due to magnetization of fuel in brake power is $3.18 \%$ when compared with BD20.

\section{Brake Specific Fuel Consumption:}

Variation in brake specific fuel consumption with load is shown in figure 6. It is seen from figure 6 that specific fuel consumption increases with the use of BD20 as fuel in place of diesel because engine fuel throttle opening increases while supplying biodiesel blends. It may be due to the lower calorific value of biodiesel fuel and its blends. BSFC was reduced with the use of magnetization of fuel because the magnetization of hydrocarbon arranges the fuel molecules in a dispersed manner and provides greater space for reacting oxygen. Hence increasing the quality of combustion and energy output from combustion.

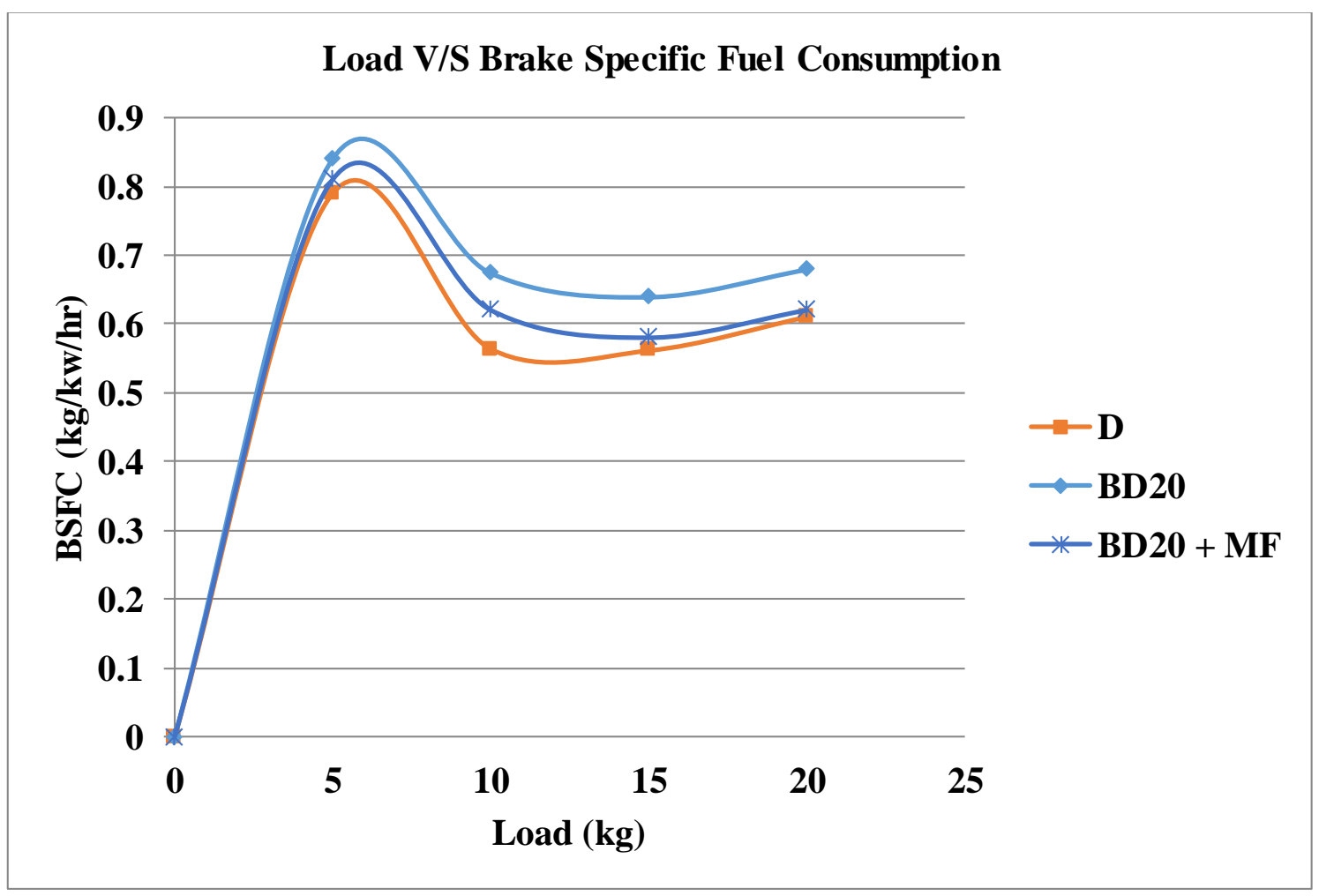

Figure 6 Variation in Brake Specific Fuel Consumption with load.

From figure 6, the maximum increase in brake specific fuel consumption with the use of BD20 is $19.52 \%$ at medium load as compared that with diesel. This increase is brought down using MF up to $10.03 \%$ as compared that with diesel. As compared to BD20 without a magnetic field, the use of MF reduces the BTE up to $7.94 \%$ at medium load and $9.19 \%$ at higher load. 
Brake Thermal Efficiency:

Figure 7 shows the variation between load and brake thermal efficiency with various conditions. The ratio of power output and total heat input is called the brake thermal efficiency. It can be seen from figure 7 that BTE reduces with the use of BD20 at all loads and with the additional use of MF, BTE again increases. BTE is a quantity that shows the power output per unit energy input, which is lower with biodiesel as a fuel. This reduction may be due to the higher density and higher viscosity of biodiesel; higher values of these properties make the energy release from fuel a little tough, while magnetization of fuel improves these qualities of fuel hence increasing BTE.

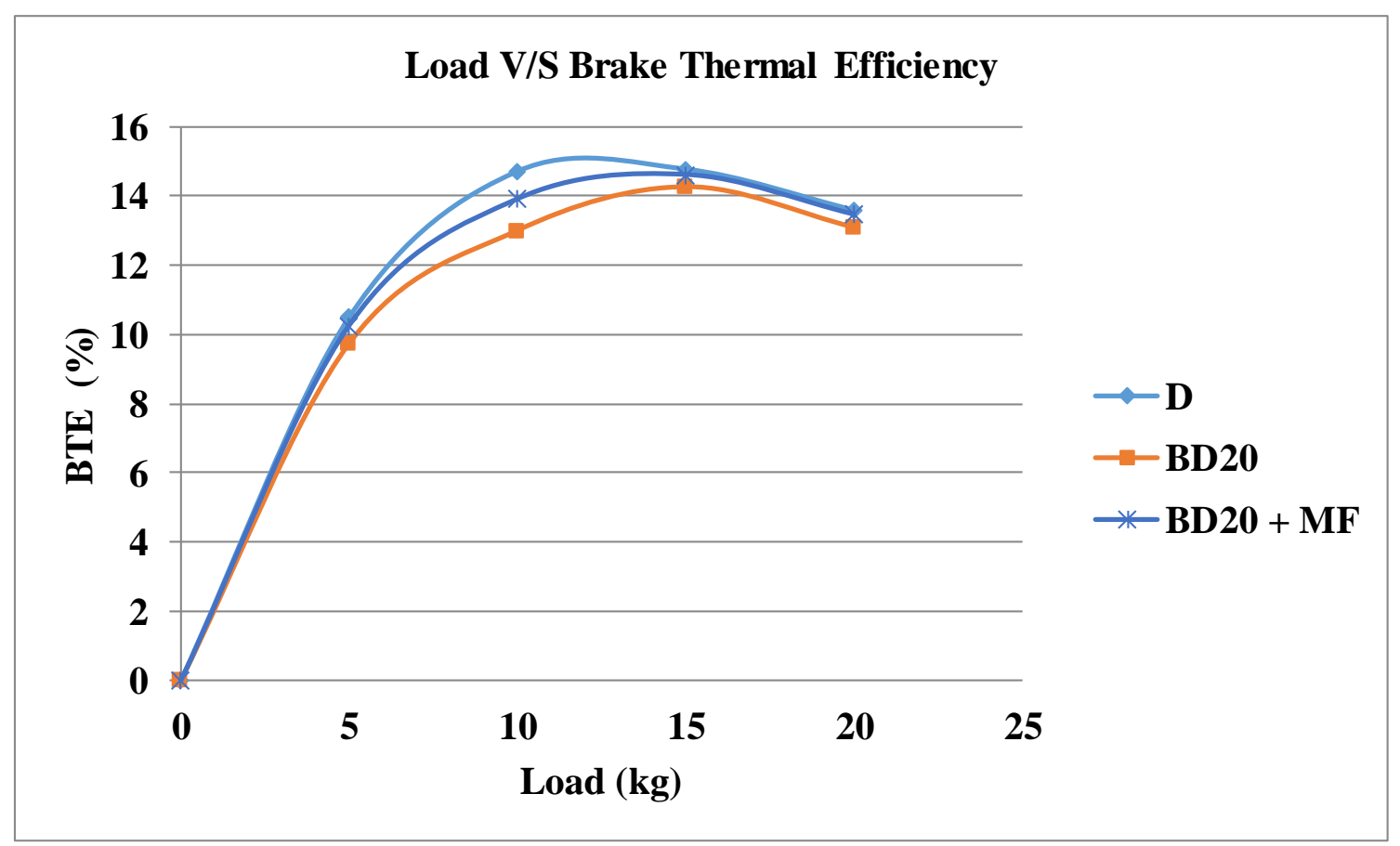

Figure 7 Variation in Brake Thermal Efficiency with Load

Figure 7 shows that the maximum decrease in brake thermal efficiency, with biodiesel as fuel, is $11.60 \%$ as compared that with diesel, additional use of MF brought down this decrease to $5.39 \%$. When a comparison has been done between BD20 and BD20+MF then there is an increase of $7.02 \%$ in BTE at a higher load.

\section{Emissions}

In the present investigation under various conditions like the use of $100 \%$ diesel (D), use of BD20, and use of BD20 with the magnetic field (BD20+MF), the main emissions considered are Carbon Mono Oxide (CO), Carbon Di Oxide (CO2), Oxides of Nitrogen (NOx), Unburnt 
Hydrocarbon (HC) and smoke. Variations in outflow quantity in each of these all some conditions are described in detail as below.

\section{Carbon Mono Oxide:}

Carbon mono oxide is a very toxic gas, which uses atmospheric oxygen to build itself. A higher quantity of $\mathrm{CO}$ in the atmosphere can cause breathing problems to humans and sometimes death also. As biodiesel has been used in a diesel engine as fuel in place of diesel, the emission of carbon monoxide from the vehicle outlet decreases, as can be seen in figure 8 . The temperature of burning gases is increased with the use of biodiesel because of higher oxygen present in the fuel and hence provide favorable conditions for complete combustion, thus reducing the emission of $\mathrm{CO}$. Emission of $\mathrm{CO}$ further reduces when magnetized fuel has been used.

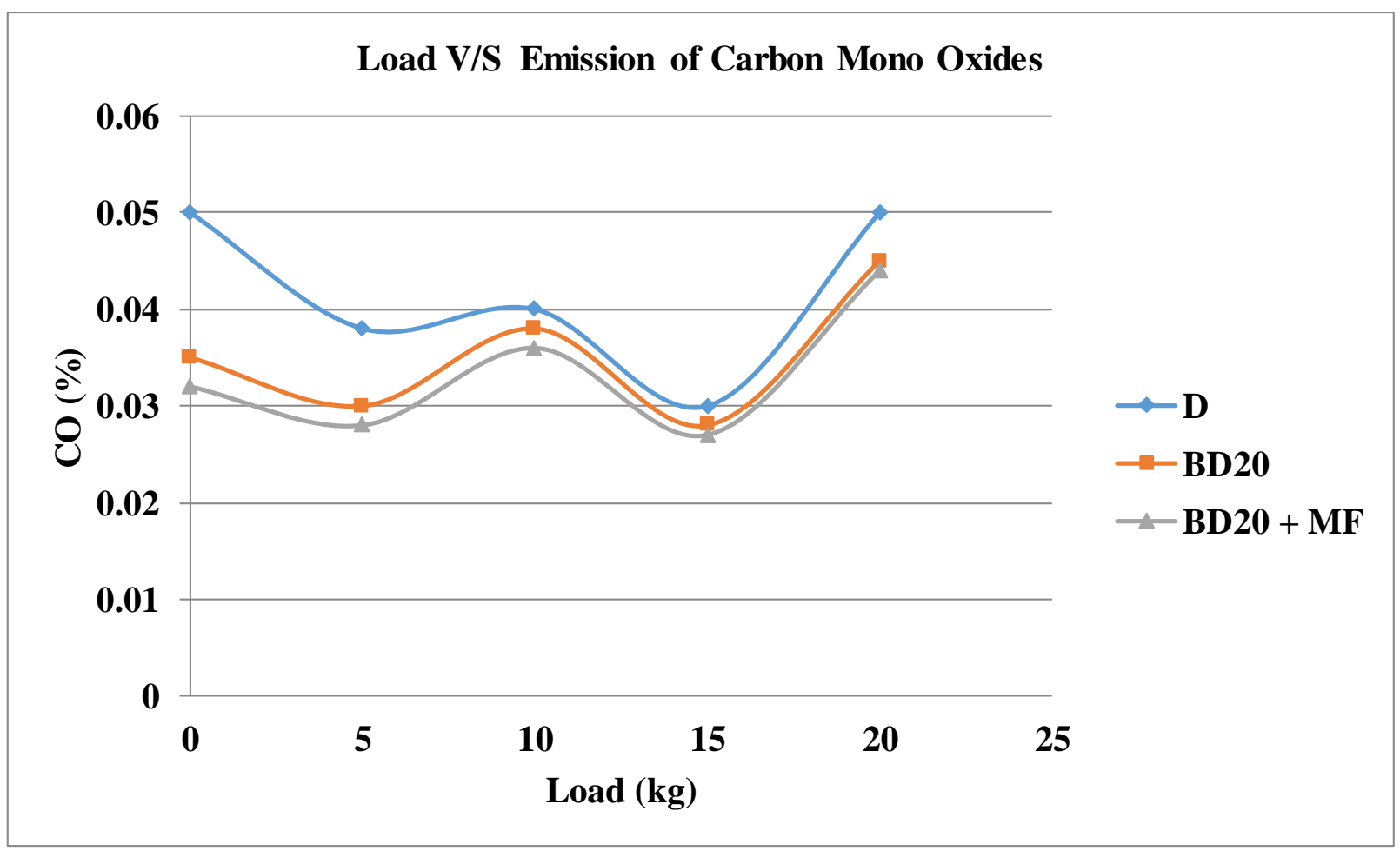

Figure 8 Variation in Emission of $\mathrm{CO}$ with load

From figure 9, the maximum decrease noticed in the emission of $\mathrm{CO}$ with the use of biodiesel is $30 \%$ as compared with the use of diesel as fuel low load. When magnetized BD20 is used, this decrease in emission of $\mathrm{CO}$ becomes $36 \%$ as compared that with diesel. When comparison has been done between BD20 and BD20+MF, then this decrease in emission of $\mathrm{CO}$ is $8.57 \%$ at low load with BD20+MF as fuel.

Oxides of Nitrogen: 
Oxides of Nitrogen are also gas which is harmful to humans if present in quantity is higher than a safe level. Biodiesel has been used in a diesel engine as an alternate, clean, and green source of energy, but with some limitations in performance and emission parameters. Being a green alternative source of energy, it emits more NOx than diesel, when used as fuel in the diesel engine. The reason behind the higher NOx emission is the higher oxygen content in the biodiesel fuel during combustion. The decrease in NOx emission with the magnetization of fuel is due to the realignment of fuel, which in turn arranges the oxygen to react with maximum atoms of carbon and hydrogen.

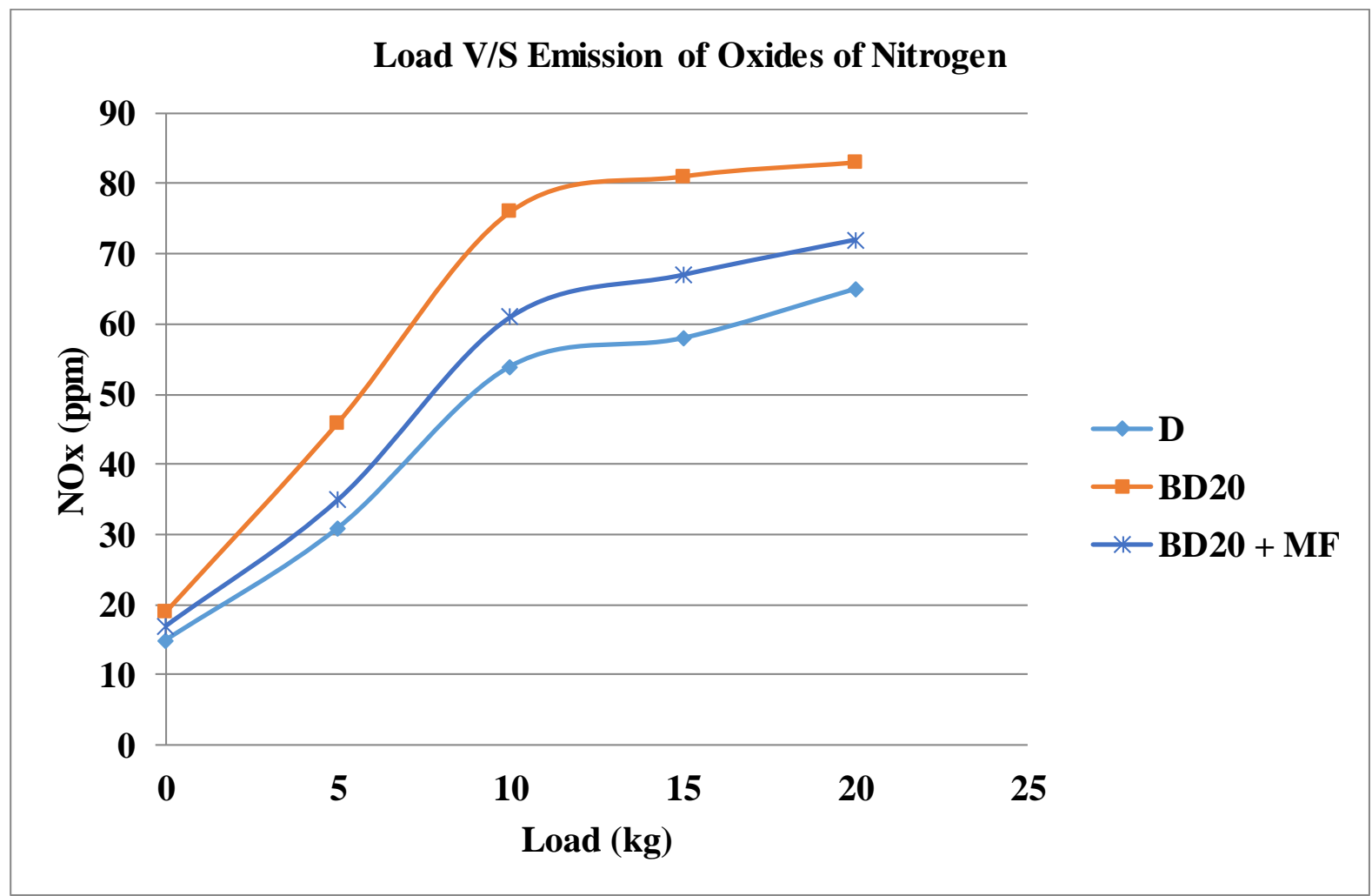

Figure 9 Variation in emission of NOx with load

From figure 9 emission of NOx increases with the use of BD20 at all loads. Emission of NOx is lower with the use of BD20+MF than the use of BD20 as fuel in diesel. A maximum increase of $48.38 \%$ at lower load has been noticed with BD20 as fuel. This increase in the emission of NOx at lower load has been brought down to $12.90 \%$ when fuel is magnetized (BD20+MF). A maximum decrease of $23.91 \%$ in the emission of NOx has been noted when BD20+MF was used as fuel, compared that with BD20 only.

\section{Un-burnt Hydrocarbon:}

The un-burnt hydrocarbon can deposit in various parts of an engine and can cause difficulty in functioning of the engine. It can be seen from figure 10 that, when biodiesel has been used as 
fuel then the emission of un-burnt hydrocarbon decreases. The main reason for the production of un-burnt hydrocarbon is the incomplete combustion of fuel. When biodiesel is used as fuel, then combustion quality improves due to higher oxygen content, thus reducing the production of HC. HC emission further reduces with the use of magnetized biodiesel at all loads because of improved properties of fuel and arrangements of fuel atoms.

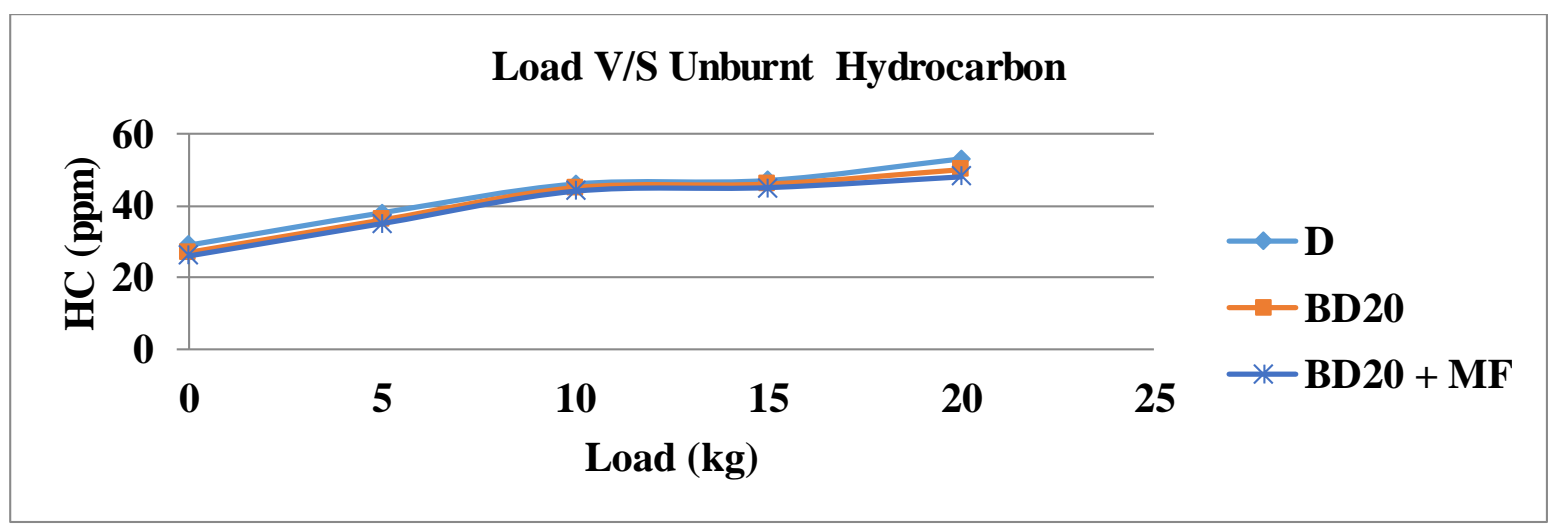

Figure 10 Variation in emission of Un-burnt Hydrocarbon with load

From figure 10, a maximum decrease of $6.89 \%$ in the outflow of $\mathrm{HC}$ has been noted with BD20 at lower load when compared that with diesel. This decrease brought down to $10.34 \%$ when biodiesel is magnetized. The decrease in HC with BD20+MF is $3.70 \%$ as compared to that with BD20.

\section{Carbon Di Oxide:}

As a result of combustion, carbon dioxide is a gas that is emitted from the exhaust of the internal combustion engine. From figure 11 it can be seen that $\mathrm{CO}_{2}$ emission increases slightly with the use of biodiesel as well as with the magnetization of biodiesel. Complete combustion produces $\mathrm{CO}_{2}$, while incomplete combustion produces $\mathrm{CO}$. Degree of complete combustion increases due to the higher content of oxygen in biodiesel and it further increases with the use of magnetized biodiesel, hence increasing the $\mathrm{CO}_{2}$ emission. 


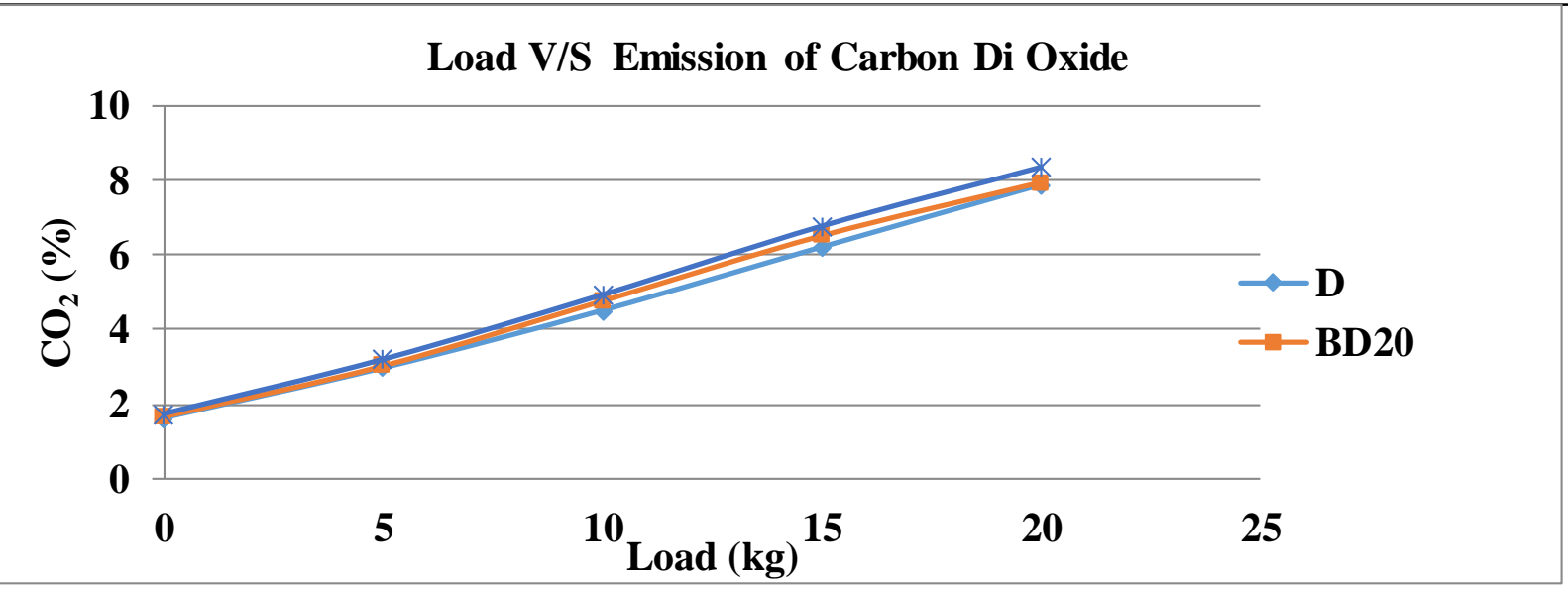

Figure 11 Variation in the emission of Carbon Di Oxide

From figure 11 it can be seen that a maximum increase of $5.53 \%$ at medium load has been noted with BD20 as compared with that of diesel. When BD20+MF is used as fuel, then at medium load $\mathrm{CO}_{2}$ emission is $3.56 \%$ higher than that with $\mathrm{BD} 20$.

Smoke:

Figure 12 shows the variation in smoke outflow at various loads with various conditions. Smoke is also not desired in the atmosphere; a higher quantity of smoke is harmful to the environment and can cause breathing problems also. From figure 12 it can be seen that smoke outflow has been reduced slightly with the use of biodiesel as fuel due to poor soot oxidation property and higher oxygen content. Smoke outflow further reduces when fuel has been magnetized.

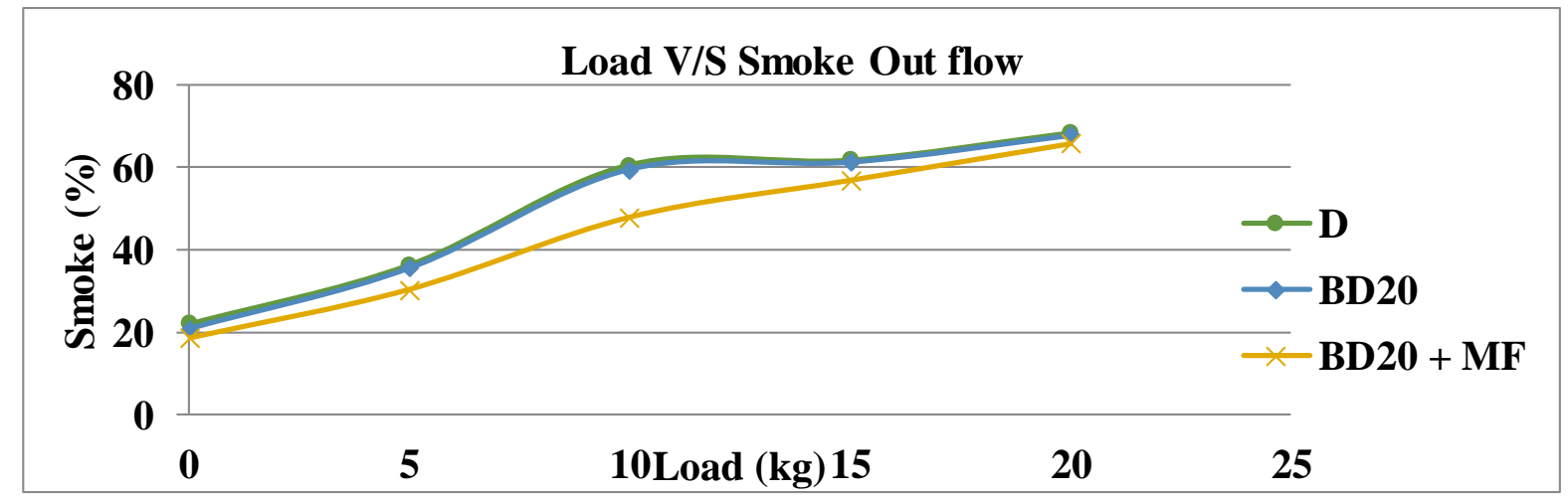

Figure 12 Variation in Smoke emission with Load

It is noted from figure 12 that, with BD20 as fuel, smoke emission is 5.6\% lower than that with diesel at lower load, and with BD20+MF smoke emission is $20.93 \%$ lower than that with diesel at medium load. With BD20+MF, a maximum decrease of $19.53 \%$ in smoke emission has been noted as compared to that with BD20. 


\section{CONCLUSION}

Significant effects of magnetized fuel on the performance and emission parameters of the internal combustion engine have been observed. Following conclusions can be made based on various results and discussion. Chemical properties and molecular structure of biodiesel have been affected by the magnetization of biodiesel, which further affects the performance and emission parameters of the internal combustion diesel engine. Applying a magnetic field of 4000 Gauss decreases the density of biodiesel by $1.605 \%$ and increases calorific value by 1.139\%.With BD20+MF, maximum reductions in BSFC, NOx, CO, HC, and smoke are $9.19 \%, 23.91 \%, 8.57 \%, 3.70 \%, 19.53 \%$ respectively as compared with that BD20.With $\mathrm{BD} 20+\mathrm{MF}$, the maximum increase in emission of $\mathrm{CO} 2$ is $5.94 \%$ as compared with that BD20 at a lower load.

\section{REFERENCES}

Wahhab, H.A.A., Al-Kayiem, H.H., Aziz, A.R.A., \& Nasif, M.S. 2017. Survey of invest fuel magnetization in developing internal combustion engine characteristics. Renewable and $\begin{array}{lllll}\text { Sustainable } & \text { Energy } & \text { Reviews. } & \text { vol. } & \text { 1392-1399. }\end{array}$ https://doi.org/10.1016/j.rser.2017.05.121

Sahoo, R.R. \& Jain, A. 2019. Experimental analysis of nanofuel additives with magnetic fuel conditioning for diesel engine performance and emissions. Fuel, vol. 236, pp. 365-372. https://doi.org/10.1016/j.fuel.2018.09.027

Thiyagarajan, S., Geo, V.E., Martin, L.J., \& Nagalingam, B. 2018. Effect of electrochemical conversion of biofuels using ionization system on $\mathrm{CO} 2$ emission mitigation in CI engine along with post-combustion system. Fuel Processing Technology, vol. 173, pp. 21-29. https://doi.org/10.1016/j.fuproc.2018.01.004

Barna, L. \& Lelea, D. 2017. The influence of magnetic field on low pressure injection of oxyhydrogen in turbocharged compression ignition engines. Procedia Engineering; vol. 181, pp. 718-724. https://doi.org/10.1016/j.proeng.2017.02.456

Faris, A.S., Al-Naseri, S.K., Jamal, N., Isse, R., Abed, M., Fouad, Z., Kazim, A., Reheem, N., Chaloob, A., Mohammad, H., Jasim, H., Sadeq, J., Salim, A. \& Abas, A. 2012. Effects of magnetic field on fuel consumption and exhaust emissions in two-stroke engine. Energy Procedia, vol. 18, pp. 327-338. https://doi.org/10.1016/j.egypro.2012.05.044 
Pramodkumar, G., Naidu, M.K., Sandeep, J.V., Vasupalli, R. \& Lade, P. 2017. Effect of magnetic field on the emission of single cylinder four stroke petrol engine. Advances in Automobile Engineering, pp. 6: 4. 10.4172/2167-7670.1000175

Afzal, A., Kareemullah, M., Razak, A.R.K. 2018. Production of biodiesel from various sources and comparative engine performance studies by using different biodiesel blends. .Journal of Engineering Research, Vol. 6, No. 4.

Niaki, S.R.A., Jadeh, F.G., Niaki, S.B.A., Mouallem, J. \& Madhavi, S. 2019. Experimental investigation of the effect of the magnetic field on performance, combustion and emission characteristics of a spark ignition engine. AICHE (Environmental Progress and Sustainable Energy). https://doi.org/10.1002/ep.13317

Rehim, A.A.A. \& Attia, A.A.A. 2014. Does magnetic fuel treatment affect engine's performance? SAE Technical Paper, 2014-01-1398. ISSN: 0148-7191, e-ISSN: 2688-3627. https://doi.org/10.4271/2014-01-1398 